\title{
SEMICONDUCTOR NANOWIRE MANIPULATION USING OPTOELECTRONIC TWEEZERS
}

Arash Jamshidi ${ }^{1}$, Peter J. Pauzauskie ${ }^{2}$, Aaron T. Ohta ${ }^{1}$, Pei-Yu Chiou ${ }^{1}$, Hsan-Yin Hsu ${ }^{1}$, Peidong Yang ${ }^{2,3}$ and Ming C. Wu ${ }^{1}$

${ }^{1}$ Berkeley Sensor \& Actuator Center (BSAC) and Department of Electrical Engineering and Computer Sciences, University of California, Berkeley, California 94720, USA

${ }^{2}$ Department of Chemistry, University of California, Berkeley, California 94720, USA

${ }^{3}$ Material Science Division, Lawrence Berkeley National Laboratory, Berkeley, California 94720, USA

Tel: 1-510-642-1023; Fax: 1-510-643-5817; email: arash@eecs.berkeley.edu

\begin{abstract}
We demonstrate, for the first time, the trapping and manipulation of individual $\mathrm{Si}$ nanowires by light-induced dielectrophoresis, or optoelectronic tweezers (OET). Trapping of single Si nanowires, with a diameter of $100 \mathrm{~nm}$ and length of $5 \mu \mathrm{m}$, is reported using OET with an optical power density of $100 \mathrm{~W} / \mathrm{cm}^{2}$. We show that OET can separate two adjacent $\mathrm{Si}$ nanowires and transport a single nanowire at a speed of $135 \mu \mathrm{m} / \mathrm{s}$. Array patterns of $\mathrm{Si}$ nanowires have also been demonstrated.
\end{abstract}

\section{INTRODUCTION}

Optical manipulation provides a powerful method to bridge the micro and nano worlds. Optical tweezers have been used to trap single silicon and CdS nanowires [1,2]; however, the ability of optical tweezers to perform parallel assembly is hampered by their high optical power density $\left(10^{7} \mathrm{~W} / \mathrm{cm}^{2}\right)$ and small working area (approximately $1 \mu \mathrm{m} \times 1 \mu \mathrm{m}$ ). Dielectrophoresis can trap nanowires between two fixed microelectrodes [3]; however, the trapping sites are fixed by the electrode pattern. In contrast, OET is capable of manipulating a large number of microparticles or cells over a large area [4, 5]. Previously, the smallest particles that OET could trap individually were limited to $1 \mu \mathrm{m}$ in diameter [5].

\section{THEORETICAL BACKGROUND}

\section{Device Operation Principles}

Figure 1a shows the structure of the optoelectronic tweezers (OET) device. The OET device consists of a top indium-tin-oxide (ITO) electrode and a bottom ITO-coated glass substrate topped with a 1- $\mu$ m-thick layer of photoconductive material (amorphous silicon). The liquid solution containing the dispersed nanowire sample is sandwiched between the top and bottom surfaces. An AC voltage is applied between the top and bottom ITO electrodes. A 632-nm HeNe laser is used to trap the $\mathrm{Si}$ nanowires. In the absence of the laser light, resistance of the photoconductive layer is higher than the resistance of the liquid layer and the majority of the applied voltage is dropped across the photoconductive layer. However, in the presence of the laser light, electron-hole pairs are generated in the photoconductive layer and the resistance of the photoconductive layer is reduced so that the majority of voltage is dropped across the liquid layer. The resistance of the photoconductive layer is only reduced in the area of the laser spot. Therefore, the electric field created in the liquid layer has a non-uniform profile [6]. Figure $1 b$ shows the finite-element simulation of the non-uniform electric field intensity and direction for an applied $\mathrm{AC}$ bias of $20 \mathrm{Vpp}$ at $50 \mathrm{kHz}$.

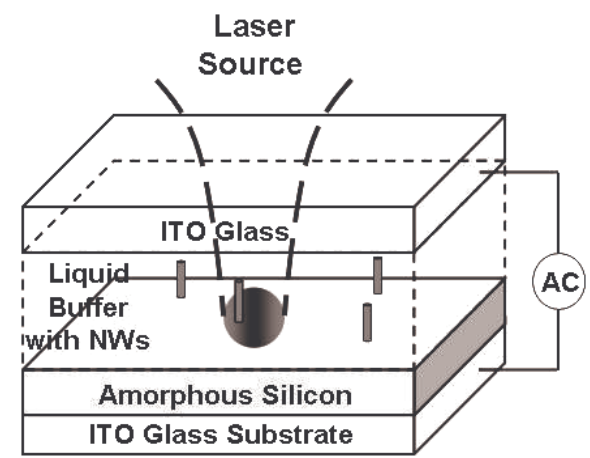

(a)

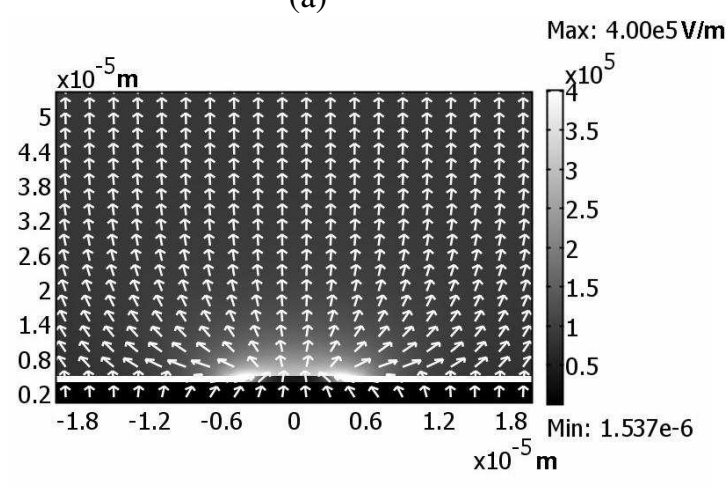

(b)

Fig.1(a) Structure of optoelectronic tweezers (OET)

1(b) Finite-element simulation of non-uniform electric field intensity and direction for an applied bias of $20 \mathrm{Vpp}$ at 50 $k H z$.

\section{Dielectrophoresis Force and Torque}

In the presence of a non-uniform electric field, a dipole moment $(p)$ is induced in the Si nanowires with unequal charges on two ends. The interaction of the induced dipole and the electric field creates a dielectrophoresis force which is either attractive or repulsive depending on the AC bias frequency and the properties of the nanowires and the liquid solution. The dielectrophoresis force can be approximated as $F=(p \cdot \nabla) E$ using the Taylor expansion of the electric field, ignoring the higher order terms [7]. 
Silicon nanowires with radius $r$ and length $l$ can be modeled as elongated ellipsoids with $\mathrm{a}, \mathrm{b}$, and $\mathrm{c}$ dimensions where $a=l / 2$ and $b=c=r$. The time-averaged dielectrophoresis force under an $\mathrm{AC}$ bias is given by:

$$
F_{D E P}=\left(\pi r^{2} l / 6\right) \varepsilon_{m} \operatorname{Re}\{K\} \nabla\left(E^{2}\right)
$$

Where $\operatorname{Re}\{K\}=\left(\varepsilon_{p}^{*}-\varepsilon_{m}^{*}\right) /\left(\varepsilon_{m}^{*}+\left(\varepsilon_{p}^{*}-\varepsilon_{m}^{*}\right) L\right)$, $\varepsilon^{*}=\varepsilon-j \sigma / \omega$, and $\mathrm{L}$ is the depolarization factor along the long axis of the nanowire [7].

The depolarization factor can be approximated by $\quad L \approx 2 r^{2}(\ln (1+f / 1-f)-2 f) / l^{2} f^{3} \quad$ where $f=\sqrt{\left(1-(2 r / l)^{2}\right.}$ [7], which results in $\mathrm{L}=1.4 \times 10^{-3}$ due to the high aspect ratio of the Si nanowires.

Using the approximate drag force for a cylindrical object moving perpendicular to its long-axis [8]:

$$
F_{\text {Drag }}=8 \pi \eta l v_{\text {drag }} /(2 \ln (l / r)-1)
$$

We can calculate the velocity of the nanowires by equating the DEP and the drag force: $F_{D E P}=F_{D r a g}$.

The DEP force experienced by $\mathrm{Si}$ nanowires is simulated using COMSOL Multiphysics finite-element software to calculate $\nabla\left(E^{2}\right)$ and the properties of the nanowires, such as radius, length, conductivity, and permittivity. The magnitude of the DEP force and nanowire speed is highly sensitive to the nanowire resistivity due to its effect on $\operatorname{Re}\{K\}$. Figures $2 \mathrm{a}$ and $2 \mathrm{~b}$ show the $\operatorname{Re}\{K\}$ and simulated DEP force and speed for nanowires of different resistivities.

In addition to the DEP force, Si nanowires also experience a torque in the presence of the non-uniform electric field which aligns the long-axis of the nanowires with the electric field.

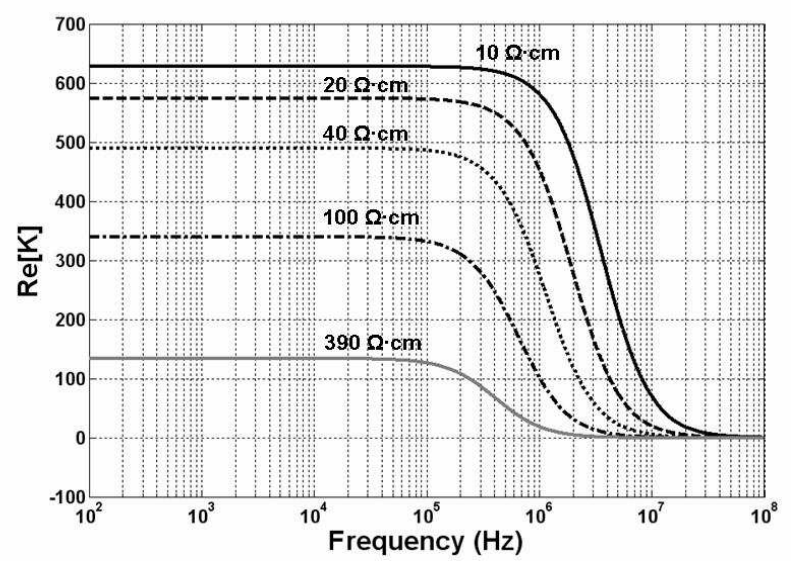

(a)

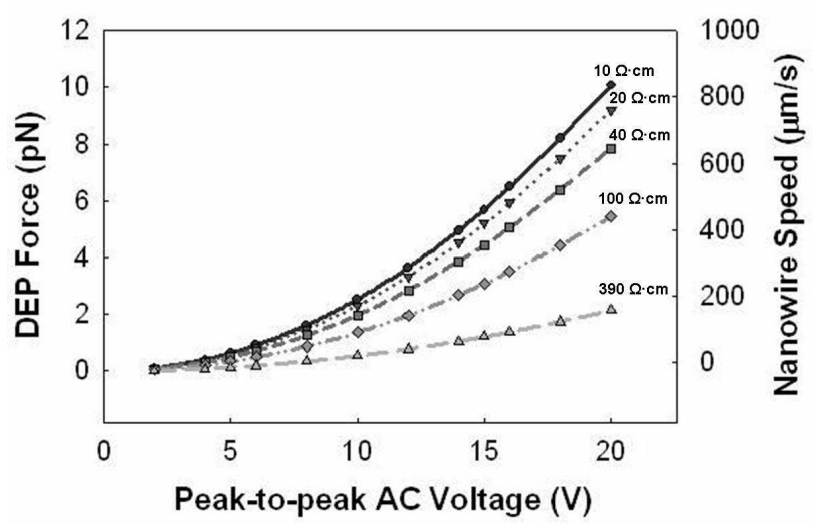

(b)

Fig.2(a) $\operatorname{Re}\{K\}$ versus frequency (b) Simulated

Dielectrophoresis-induced speed and force versus peak-to-peak AC voltage for Si NWs of various resistivities.

\section{EXPERIMENTAL RESULTS}

\section{Experimental Setup}

Figure 3 shows the experimental setup for the DEP manipulation of Si nanowires. Slightly boron doped silicon nanowires of $100 \mathrm{~nm}$ diameter and approximately $5 \mu \mathrm{m}$ length were diluted in a $1.5 \mathrm{mS} / \mathrm{m}$ conductivity solution of DI water and $\mathrm{KCl} .3 \mu \mathrm{L}$ of the sample was introduced into the OET device. A portion of the Si nanowires experienced Brownian motion in the solution while others adhered to the surface of the OET chip. A HeNe laser with a power of 100 $\mu \mathrm{W}$ at the OET surface was used to trap silicon nanowires. A 40X objective lens is used to focus the laser, the resulting optical spot size is $10 \mu \mathrm{m}$ (FWHM). AC Voltages of 5, 8, $10,12,15,18$, and 20 Volts peak-to-peak at frequencies of $50 \mathrm{kHz}$ and $100 \mathrm{kHz}$ were applied to the OET device. Dark field observations were made using an Olympus BX51M microscope, and images were captured using a CCD camera.

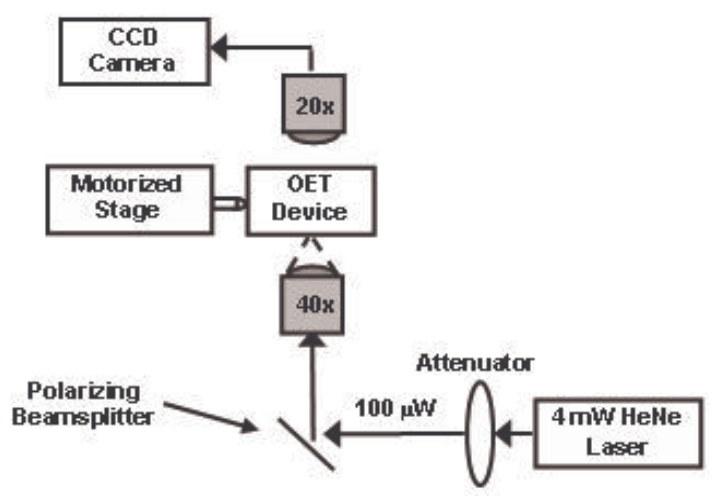

Fig.3 Experimental setup for manipulation of silicon nanowires. A 4-mW HeNe laser was attenuated to $100 \mu W$ and focused onto the OET chip for manipulation of nanowires using a $40 X$ objective lens. 


\section{Synthesis}

Silicon nanowire samples were grown by chemical vapor deposition (CVD) of silicon tetrachloride $\left(\mathrm{SiCl}_{4}\right)$ onto a silicon wafer, which was gold-coated by e-beam evaporation of $\mathrm{Au}$, in a $850{ }^{\circ} \mathrm{C}$ furnace for approximately 30 minutes. Figures $4 \mathrm{a}$ and $4 \mathrm{~b}$ show the transmission electron microscopy (TEM) and scanning electron microscopy (SEM) images of the Si nanowire samples.

The TEM images reveal that the nanowire samples do not have any noticeable gold catalyst residues on their surface. In addition, there is a very thin layer of oxide, a few angstroms in size, on the surface of the nanowires. Using the SEM images, we can characterize the radius and length of the $\mathrm{Si}$ nanowires. On average, the nanowires have a radius of approximately $100 \mathrm{~nm}$ and a length of $5 \mu \mathrm{m}$. The change in the growth direction of the nanowires, visible in the images, is due to cutting off the gas flow at the end of the growth process.

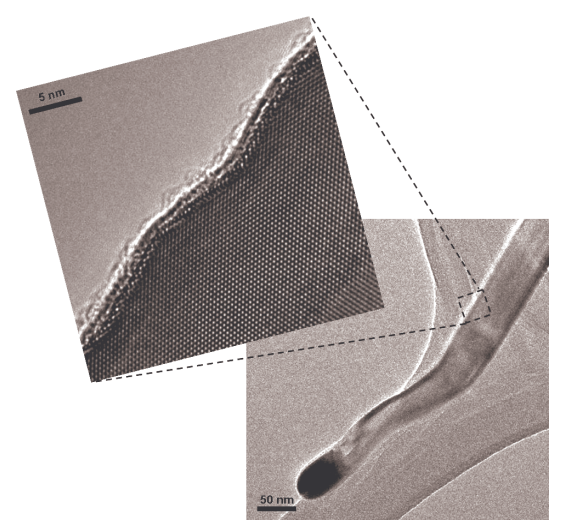

(a)

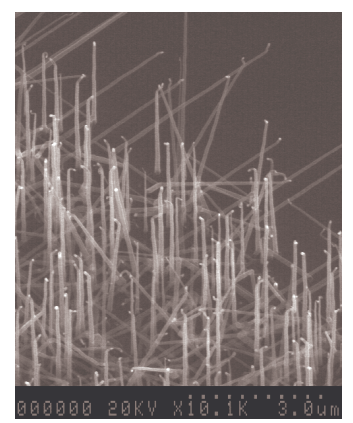

(b)

Fig.4(a) TEM images of the Si nanowire samples at 2.9K and $41 \mathrm{~K}$ magnifications. (b) SEM image of Si nanowire samples at $10.1 \mathrm{~K}$ magnification.

\section{DEP Manipulation of Silicon Nanowires}

Upon application of voltage across the OET device, the long-axis of the nanowires aligned with the electric field in the liquid layer. After turning on the laser, Si nanowires experienced an attractive force towards the illuminated area. Figure 5 shows the trapping of an individual $\mathrm{Si}$ nanowire. Before time $0 \mathrm{~s}$, the applied voltage was off, and the nanowire underwent Brownian motion. At time $0 \mathrm{~s}$ the voltage was turned on, the nanowire aligned with the electric field and it took $2 \mathrm{~s}$ for the nanowire to reach the laser.

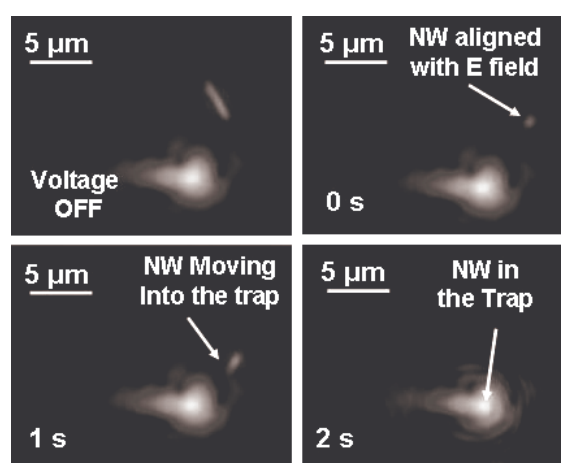

Fig.5 Trapping of an individual Si nanowire. A voltage of $15 \mathrm{Vpp}$ at $100 \mathrm{kHz}$ is applied to the OET device.

\section{Speed and Trapping Radius Measurements}

To quantify the trapping of the Si nanowires, the maximum speed of the nanowires was measured by moving the stage of the microscope in relation to the optical pattern via a ESP-300 Newport motorized actuator controller, and a LTA-HL motorized actuator. Figure 6 shows the measured maximum speed of the nanowires versus the applied AC voltage. Speeds of $135 \mu \mathrm{m} / \mathrm{s}$ were observed with an applied voltage of $20 \mathrm{Vpp}$. In addition, the trapping radius of OET for Si nanowires, defined as the maximum distance that the laser spot can be from the nanowire, yet still be able to overcome the Brownian motion, was also measured. Figure 7 shows the measured trapping radius as a function of AC voltage. The trapping radius of approximately $122 \mu \mathrm{m}$ was achieved at $20 \mathrm{Vpp}$. As expected from the simulations, both the maximum speed and the trapping radius show a quadratic dependence on the magnitude of the AC voltage, due to an increase in the DEP force. However, the magnitudes of the simulated and measured speeds are harder to compare due to the variations in size and resistivity of the Si nanowires. Based on simulation results (Fig. 2), a Si nanowire with $390 \Omega \cdot \mathrm{cm}$ resistivity would experience speeds with magnitudes comparable to the experimental results.

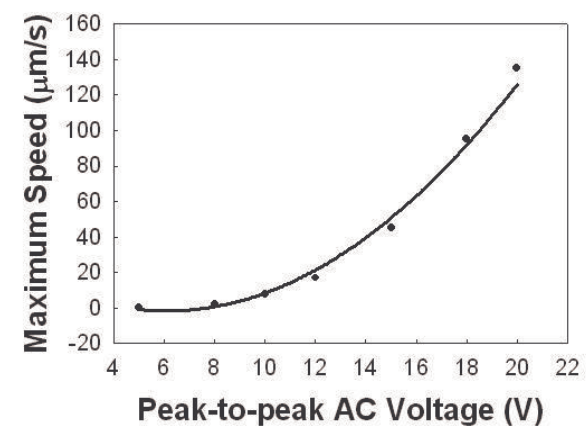

Fig.6 Maximum speed measurements of silicon nanowires versus the applied AC voltage. The solid line is a fitted quadratic curve to the experimental data. 


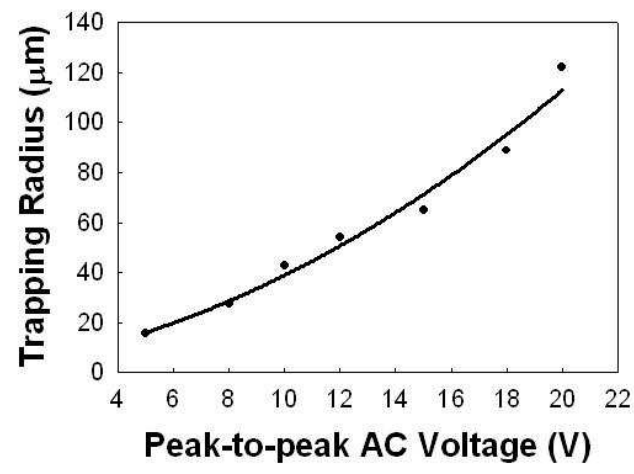

Fig.7 Maximum trapping radius of silicon nanowires versus the applied $A C$ voltage. The solid line is a fitted quadratic curve to the experimental data

Nanowires within a trapping radius can still be trapped individually by controlling the scanning speed of the laser spot. Figure 8 shows the separation of two adjacent $\mathrm{Si}$ nanowires.
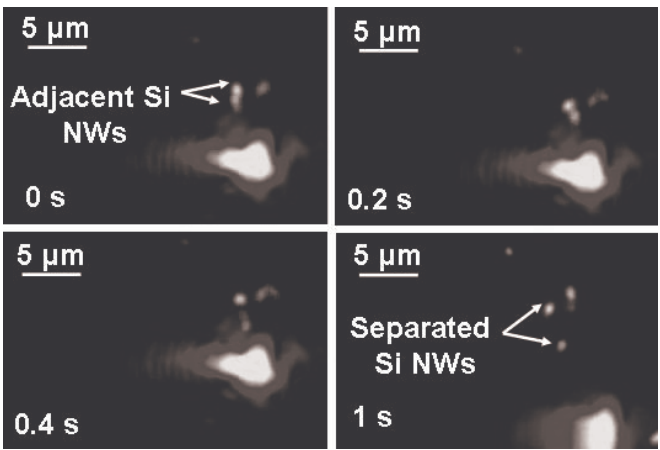

Fig.8 Separation of two adjacent Si nanowires. An AC voltage of $10 \mathrm{Vpp}$ at $100 \mathrm{kHz}$ is applied to the OET device.

\section{Nanowire Assembly}

The flexibility of the OET device introduces the ability to arrange the Si nanowires into arbitrary structures. Figures 9a and $9 \mathrm{~b}$ show the arrangement of $\mathrm{Si}$ nanowires into arrays of different shapes. The nanowires appear as circular dots, since they were aligned to the vertical electric field. The white dots in the background are the nanowires that have adhered to the surface of the OET chip. A spherical lens is used to create a line laser pattern for movement of $\mathrm{Si}$ nanowires in arrays of 2 or 3 .

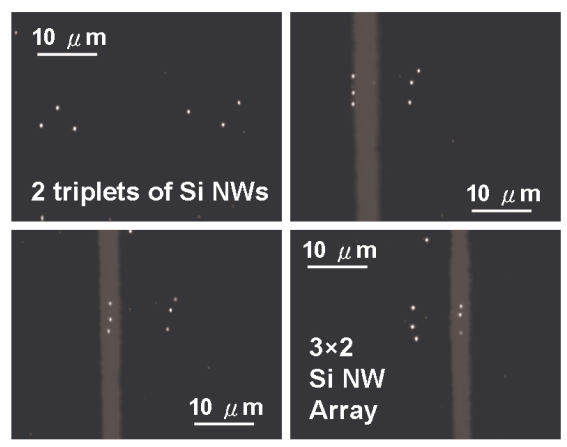

(a)

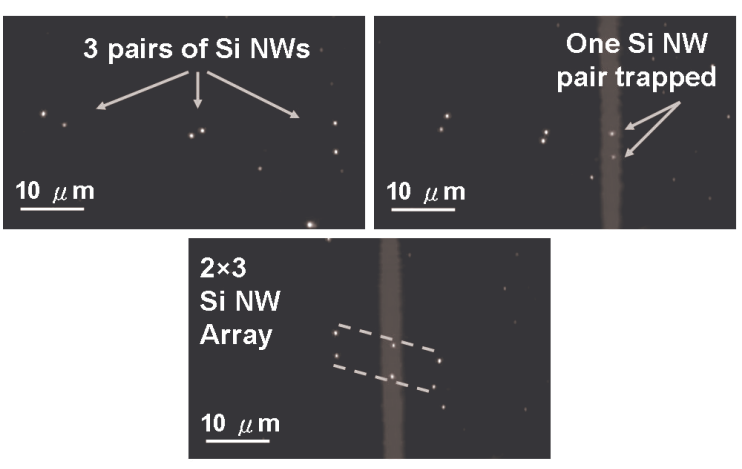

(b)

Fig.9 Arrangement of silicon nanowires into (a) a triangular structure and $3 \times 2$ array $(b)$ a $2 \times 3$ array. Silicon nanowires were aligned vertically with the electric field

\section{CONCLUSION}

The flexibility of the optoelectronic tweezers device, low required optical power intensity, and large working area makes OET a very attractive tool for the manipulation of nanowires. In this paper, the trapping and assembly of $\mathrm{Si}$ nanowires using light-induced dielectrophoresis is demonstrated for the first time. A maximum velocity of 135 $\mu \mathrm{m} / \mathrm{s}$ and a trapping radius of $122 \mu \mathrm{m}$ are achieved using this method.

\section{ACKNOWLEDGEMENT}

This project is supported by Berkeley Sensor and Actuator Center (BSAC) and Institute for Cell Mimetic Space Exploration (CMISE), a NASA URETI. AJ and PJP would like to thank Miki Kunitake for preparation of Si nanowire samples. PJP thanks the NSF for a graduate student research fellowship. The authors thank the National Center for Electron Microscopy for use of their facilities.

\section{REFERENCES}

[1] P. J. Pauzauskie, et al., Nature Materials, vol. 5, February 2006

[2] R. Agarwal, et al, Optics Express, vol. 13, No. 22, 31 October 2005

[3] P. A. Smith, et al., Applied Physics Letters, vol. 77, Number 9, 28 August 2000

[4] P. Y. Chiou, A. T. Ohta, M. C. Wu, Nature, vol. 436, pp. 370-372, 2005.

[5] P. Y. Chiou, et al, $17^{\text {th }}$ IEEE International Conference on Micro Electro Mechanical Systems, pp.21-24, 2004

[6] P. Y. Chiou, et al, Proceeding of IEEE/LEOS International Conference on Optical MEMS and Their Applications, pp. 8-9, 2003

[7] T. B. Jones, Electromechanics of Particles (Cambridge: Cambridge University Press), 1995

[8] H. Morgan and N. Green, AC Electrokinetics: colloids and nanoparticles (Research Studies Press Ltd.), 2003 